\title{
Control of multiferroic domains by external electric fields in $\mathrm{TbMnO}_{3}$
}

\author{
J. Stein ${ }^{1}$, M. Baum $¥$, S. Holbein ${ }^{1}$, V. Hutanu ${ }^{2,3}$, A. C. \\ Komarek $^{1}$, and M. Braden ${ }^{1}$ \\ ${ }^{1}$ II. Physikalisches Institut, Universität zu Köln, Zülpicher Straße 77, D-50937 Köln, \\ Germany \\ ${ }^{2}$ RWTH Aachen University, Institut für Kristallographie, D-52056 Aachen, Germany \\ ${ }^{3}$ Forschungszentrum Jülich GmbH, Jülich Centre for Neutron Science at MLZ, \\ D-85747 Garching, Germany \\ E-mail: braden@ph2.uni-koeln.de
}

31 March 2015

\begin{abstract}
The control of multiferroic domains through external electric fields has been studied by dielectric measurements and by polarized neutron diffraction on singlecrystalline $\mathrm{TbMnO}_{3}$. Full hysteresis cycles were recorded by varying an external field of the order of several $\mathrm{kV} / \mathrm{mm}$ and by recording the chiral magnetic scattering as well as the charge in a sample capacitor. Both methods yield comparable coercive fields that increase upon cooling.
\end{abstract}

PACS numbers: $61.05 . F-75.85 .+t$ 75.25.-j

Submitted to: J. Phys. C: Solid State Phys.

$\ddagger$ present address: Fraunhofer INT, Euskirchen, Germany 


\section{Introduction: chiral domains in multiferroic compounds}

Multiferroics continue attracting strong interest due to their application potential in data storage and processing [1, 2]. In particular the more recently discovered multiferroic materials, in which the ferroelectric polarization is directly coupled to the magnetic order [2], are promising, because they allow one to control the magnetic structure through an external electric field. In these materials the magnetic structure is rather complex with non-collinear and frequently chiral arrangement of magnetic moments. Such a non-collinear arrangement can induce a local electric dipole through the inverse Dzyaloshinski-Moriya mechanism, $\vec{P} \propto \vec{r}_{i j} \times\left(\vec{S}_{i} \times \vec{S}_{j}\right)$, where $\vec{r}_{i j}$ is the connecting vector between two neighboring spins $\vec{S}_{i}$ and $\vec{S}_{j}[3]$. For example in a cycloid these local dipoles add up to macroscopic ferroelectric polarization. This mechanism perfectly describes the multiferroic phases in many of the transition-metal oxides multiferroics such as $\mathrm{TbMnO}_{3}$ [4], $\mathrm{Ni}_{3} \mathrm{~V}_{2} \mathrm{O}_{8}$ [5] and $\mathrm{MnWO}_{4}$ [6]. The inverse Dzyaloshinski-Moriya interaction is not the only possibility to induce ferroelectric polarization through a canted spin arrangement [7, 8. For example for lower symmetry such as in monoclinic systems polarization may also appear parallel to $\vec{S}_{i} \times \vec{S}_{j}$, which applies to the multiferroic phases in $\mathrm{CuFeO}_{2}$ [7, 9], $\mathrm{CuCrO}_{2}$ [10], $\mathrm{RbFe}\left(\mathrm{MoO}_{4}\right)_{2}$ [11] and $\mathrm{NaFeSi}_{2} \mathrm{O}_{6}$ [12].

In the multiferroic phase there are at least two different electric domains corresponding to the direction of the ferroelectric polarization. These electric domains are coupled with the magnetic structure, which is chiral in most cases. A cycloid does not exhibit a scalar chirality but a vector chirality, which can be directly associated with the ferroelectric polarization. Therefore, the magnetoelectric coupling in the multiferroic phase allows one to control the chiral magnetism with the aid of an external electric field. In a future application one can add an exchange bias coupled ferromagnetic layer on top of the multiferroic compound in order to read the multiferroic state [13]. Studying the multiferroic domains in a bulk material is, however, more difficult and requires a microscopic technique such as second harmonic generation [14, 15] or polarized neutron diffraction [16.

Polarized neutron diffraction is ideally suited to study chiral magnetism [16]. This can be easily understood, because the neutron is a chiral object itself when its magnetic moment and momentum are parallel. In general magnetic neutron diffraction measures the Fourier component of the magnetization distribution, $\vec{M}$, but only the components perpendicular to the scattering vector, $\vec{Q}$, contribute, $\vec{M}_{\perp}$ with $\left|\vec{M}_{\perp}\right|^{2}=\left|M_{y}\right|^{2}+\left|M_{z}\right|^{2}$. Here we use the common coordinate system in polarized neutron scattering experiments $-\vec{x} \| \vec{Q}, \vec{z}$ vertical to the scattering plane, and $\vec{y}=\vec{z} \times \vec{x}$. The chiral magnetic scattering is defined as $M_{\chi}^{2}=-i\left(\vec{M}_{\perp} \times \vec{M}_{\perp}^{*}\right)_{x}$ as it can be illustrated by considering an ideal helix. For such ideal helix and $\vec{Q}$ parallel to the propagation vector $M_{\chi}^{2}$ is maximal and of the same size as $\left|\vec{M}_{\perp}\right|^{2}$. Using spherical polarization analysis one may analyze the full polarization matrix, i.e. analyzing the neutron polarization in the outgoing beam parallel to $x, y$, or $z$ for any polarization in the incoming beam. The intensity in such a channel, $\sigma_{u v}$ with $u, v=x, y$, or $z$, can be recorded for up and down polarization, 
respectively, resulting in four values in each channel. We denote the down polarization by an overbar. For the longitudinal cases, $\sigma_{x x}, \sigma_{y y}$ and $\sigma_{z z}$, one may distinguish spinflip (SF) and non-spin-flip (NSF) processes. The chiral components can be directly determined in various channels, most easily they are obtained in the $x x$ channels where all the magnetic scattering contributes to the SF scattering. For these two SF intensities one finds $\sigma_{x \bar{x}}=\vec{M}_{\perp} \cdot \vec{M}_{\perp}^{*}-i\left(\vec{M}_{\perp} \times \vec{M}_{\perp}^{*}\right)_{x}$ and $\sigma_{\bar{x} x}=\vec{M}_{\perp} \cdot \vec{M}_{\perp}^{*}+i\left(\vec{M}_{\perp} \times \vec{M}_{\perp}^{*}\right)_{x}$. In one case the chiral contribution adds to $\left|\vec{M}_{\perp}\right|^{2}$, while it is subtracted in the other case. In the case of the ideal helix with the propagation vector parallel to $\vec{Q}$ the total intensity will thus appear only in one of the two SF $x x$ channels. However, this applies only to a mono-domain sample, while for equal domain distribution the two $\sigma_{x \bar{x}}$ and $\sigma_{\bar{x} x}$ SF channels become again identical. Comparing these two SF channels gives thus a direct method to determine the domain distribution in a chiral magnet. [16, 17, 18, 19].

Concerning the multiferroic materials neutron polarization analysis was first applied for $\mathrm{TbMnO}_{3}$ to show that one can control the chiral domains by cooling in an external electric field [19]. Similar temperature dependent measurements were performed for $\mathrm{LiCu}_{2} \mathrm{O}_{2}$ [20] and $\mathrm{MnWO}_{4}$ [18, 21]. For $\mathrm{MnWO}_{4}$ neutron polarization analysis was also used to follow multiferroic hysteresis cycles by varying the external electric field at constant temperature and recording the chiral domain distribution [18, 22, 23].

$\mathrm{TbMnO}_{3}$ is the prototypical material for the new class of chiral multiferroic materials, because it exhibits sizable ferroelectric polarization and large magnetoelectric coupling combined with a moderately complex crystal and magnetic structure [4, 24, 25]. Magnetic order in $\mathrm{TbMnO}_{3}$ sets in at $\mathrm{T}_{\mathrm{N}}=42 \mathrm{~K}$ leading to a longitudinal spin-density wave (SDW) with a propagation vector of $\vec{k}_{i n c}=(0 \sim 0.280)$ in reduced lattice units of the Pbnm structure. Upon further cooling below $\mathrm{T}_{\mathrm{c}}=27.3 \mathrm{~K}$ a second magnetic transition occurs into an elliptical cycloid phase with nearly the same propagation vector. In the SDW phase spins are aligned parallel to the $\mathbf{b}$ direction, while they rotate in the bc plane in the cycloidal phase. This second transition is accompanied by the onset of ferroelectric order, which is perfectly explained by the inverse Dzyaloshinski-Moriya mechanism. The same mechanism also describes the rotation of the ferroelectric polarization in external magnetic fields [26], as the cycloid plane flops simultaneously from the bc to the ab plane [27. The multiferroic hysteresis loops have not been examined in detail for $\mathrm{TbMnO}_{3}$, only a single loop was studied by second harmonic generation on a thin film [28].

Here we study the control of the multiferroic domain population by application of an external electric field in $\mathrm{TbMnO}_{3}$. We directly measure the ferroelectric polarization by counting the surface charge in a capacitor and we determine the chiral domain distribution by polarized neutron diffraction. Both methods document that it is possible to switch the multiferroic domains in bulk $\mathrm{TbMnO}_{3}$ by varying the external field at constant temperature, but only sufficiently close to the ferroelectric transition. 


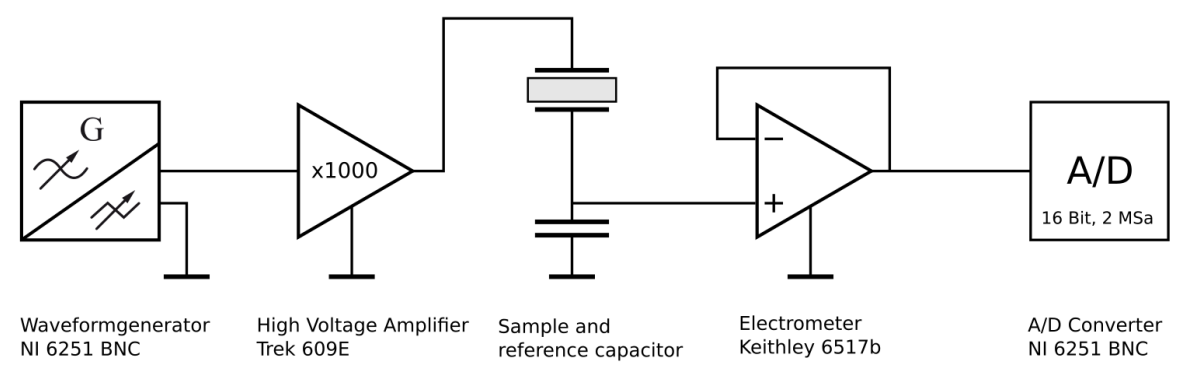

Figure 1. Scheme of the setup used for dielectric measurements of the multiferroic domains in $\mathrm{TbMnO}_{3}$. It consists of a modified Sawyer-Tower circuit.

\section{Experimental}

A large single crystal of $\mathrm{TbMnO}_{3}$ was grown with the travelling solvent floating-zone technique in a mirror furnace (Crystal Systems Incorporated FZ-T-10000-H-VI-VP). The magnetic transitions were determined by SQUID measurements on two identically grown crystals to occur at $T_{N}=42.0(2)$ and $T_{C}=27.3(2) \mathrm{K}$ in good agreement with literature [4, 24]. All crystals used in the neutron diffraction and macroscopic studies in this work were cut from the same single crystal.

Polarized neutron diffraction experiments were performed with the polarized singlecrystal diffractometer POLI-HEIDI installed at the hot source of the Forschungsreaktor München II (FRM-II). The neutron polarization and analysis is accomplished by ${ }^{3} \mathrm{He}-$ spin filters in the incoming and scattered beams [29, 30, 31]. In order to control the decay of the first filter polarization the incoming beam polarization was recorded by a transmission monitor. The polarization analysis of the scattered beam was verified by analyzing a purely nuclear Bragg reflection. The direction of the neutron polarization at the sample was controlled with the CRYOPAD setup [16].

In order to study the effect of crystal thickness and to apply an electrical field parallel to the $\mathbf{c}$ direction, two plate-shaped single-crystals were cut perpendicular to the c axis with a thickness of $3.1 \mathrm{~mm}$ and $0.9 \mathrm{~mm}$, respectively. The electric field was applied with a capacitor build from aluminum plates and nylon screws. The sample was mounted

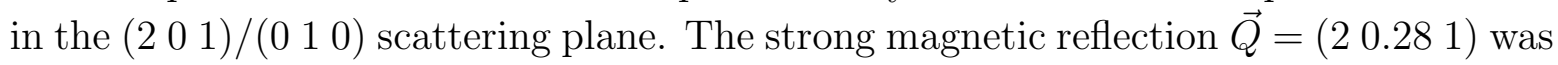
used for determining the chiral ratio. Note that a large $h$ component of the magnetic Bragg reflection is needed in this experiment, because both components of the bc cycloid must strongly contribute to the scattering, which requires that the $b$ and $c$ axes are nearly perpendicular to $\vec{Q}$. The intensity of the thin sample at this reflection was rather low, $50 \mathrm{cts} / \mathrm{s}$. The sample was cooled with a standard FRM-II type top-loading closed-cycle refrigerator, which stabilizes temperatures within $0.1 \mathrm{~K}$. The challenge of measurements with high electric fields in croygenic environments consists in fine-tuning the pressure of helium exchange gas continuously on a thin line where it is possible to apply several $\mathrm{kV} / \mathrm{mm}$ without electric discharge while the sample temperature is still controllable. 


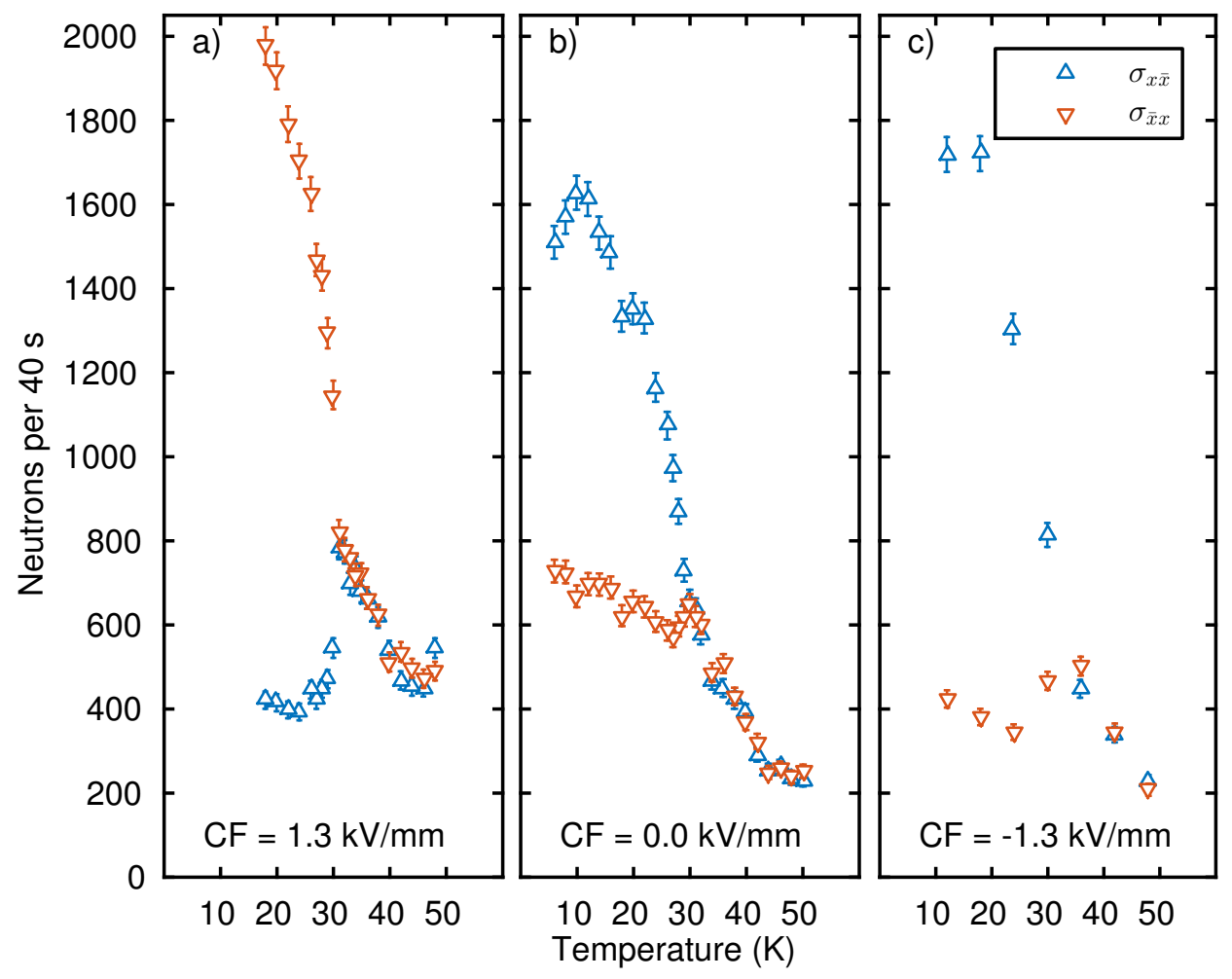

Figure 2. (Color online) The magnetic scattering at the (2 0.28 1) Bragg reflection in $\mathrm{TbMnO}_{3}$ was recorded in the two SF channels for neutron polarization along the $x$ direction. During cooling through the magnetic transitions, electric fields of $\pm 1.3 \mathrm{kV} / \mathrm{mm}$ were applied in (a) and (c), while the data in (b) were taken in zero field. The intensities of the two SF channels $\sigma_{x \bar{x}}$ and $\sigma_{\bar{x} x}$ interchange upon reversing the field, which indicates the population of opposite chiral domains.

Much efforts were needed to determine the breakdown pressure versus voltage curves before starting the experiment. A temperature offset of about $1 \mathrm{~K}$ was observed for the phase transitions measured with applied field and those reported in the literature. This offset arises from the insufficient thermal contact between the temperature sensor and the isolating sample, when the exchange gas is lowered to allow for the application of electric field. In the following we assume this temperature offset to be constant in the temperature range from 10 to $50 \mathrm{~K}$ and correct the data correspondingly. The chiral ratio was determined by measuring the two SF $\sigma_{x \bar{x}}$ and $\sigma_{\bar{x} x}$ channels as described above.

The macroscopic ferroelectric polarization was measured with a modified SawyerTower circuit, in which the surface charge is determined indirectly with an electrometer (Keithley 6517b), see Figure 1. A thin plate of $0.2 \mathrm{~mm}$ thickness and a contacted surface area of $9 \mathrm{~mm}^{2}$ was cut from the same large crystal as those used in the neutron diffraction experiments. The crystal was contacted on both sides with conductive silver paint in order to form the sample capacitor, which is studied in respect to the reference capacitor in the Sawyer-Tower circuit. We applied electric fields of up to $3 \mathrm{kV} / \mathrm{mm}$ and recorded the hysteresis loops in the quasistatic configuration. Running a full loop 


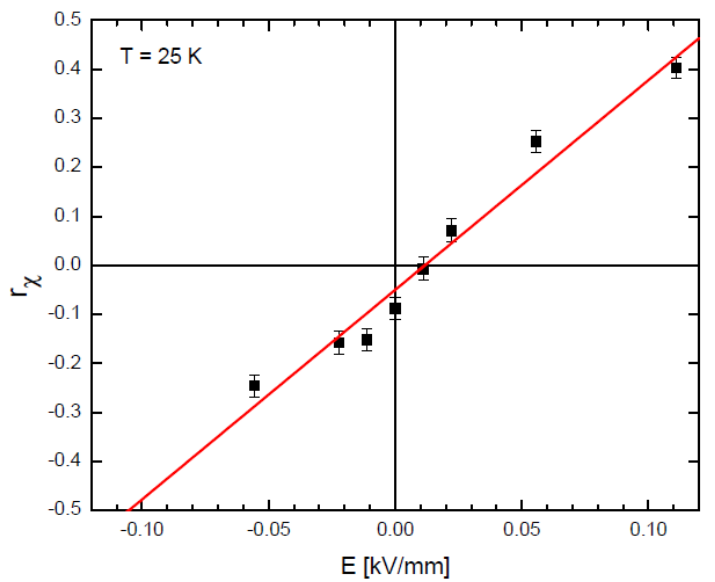

Figure 3. (Color online) Chiral ratio measured at $\mathrm{T}=25 \mathrm{~K}$ after cooling the thick sample in small electric fields from the paramagnetic $(50 \mathrm{~K})$ into the chiral magnetic phase $(25 \mathrm{~K})$. The sequence was measured with reducing and alternating field strengths. At low electric fields the chiral ratio determined at $25 \mathrm{~K}$ shows a linear dependence on the electric field applied during cooling.

corresponds to one second. The high voltage was generated by symmetric triangular waveforms which have been amplified by a factor of 1000 in a Trek 609E high voltage amplifier. In a vacuum of about $10^{-6} \mathrm{mbar}$ it was possible to apply electric fields of the order of $5 \mathrm{kV} / \mathrm{mm}$. The results are limited by finite conductivity losses, which turned out to be small. The sample capacitor was mounted on the cold finger of a closed cycle refrigerator, which allows to reach base temperatures of $5 \mathrm{~K}$. The control of temperature and the runs to record the hysteresis loops were fully automatized. Comparing several hysteresis loops no ongoing fatigue effects could be detected, but fatigue effects for an asgrown crystal cannot be excluded. Therefore, it is possible to average 4 hysteresis loops at each temperature. This procedure effectively suppresses noise which partially stems from the triboelectric effect.

\section{Results}

\subsection{Polarized neutron diffraction experiments}

In a preliminarily test the sample was cooled from $50 \mathrm{~K}$ in positive and negative electric fields of $\pm 1.3 \mathrm{kV} / \mathrm{mm}$ and in zero field, see Figure 2. The sample develops a clear preference for one chiral domain depending on the sign of the electric field. The intensity of the two spin-flip channels is interchanged by reversing the electric field. The chiral ratio, $r_{\text {chir }}=\frac{\sigma_{x \bar{x}}-\sigma_{\bar{x} x}}{\sigma_{x \bar{x}}+\sigma_{\bar{x} x}}$, was calculated from the data for cooling in positive field and roughly follows the development of the electric polarization as it has been previously reported by the same type of experiment [19]. Note that even for a mono-domain state $r_{\text {chir }}$ will deviate from \pm 1 due to the finite geometry factors in $\mathrm{TbMnO}_{3}$, which 

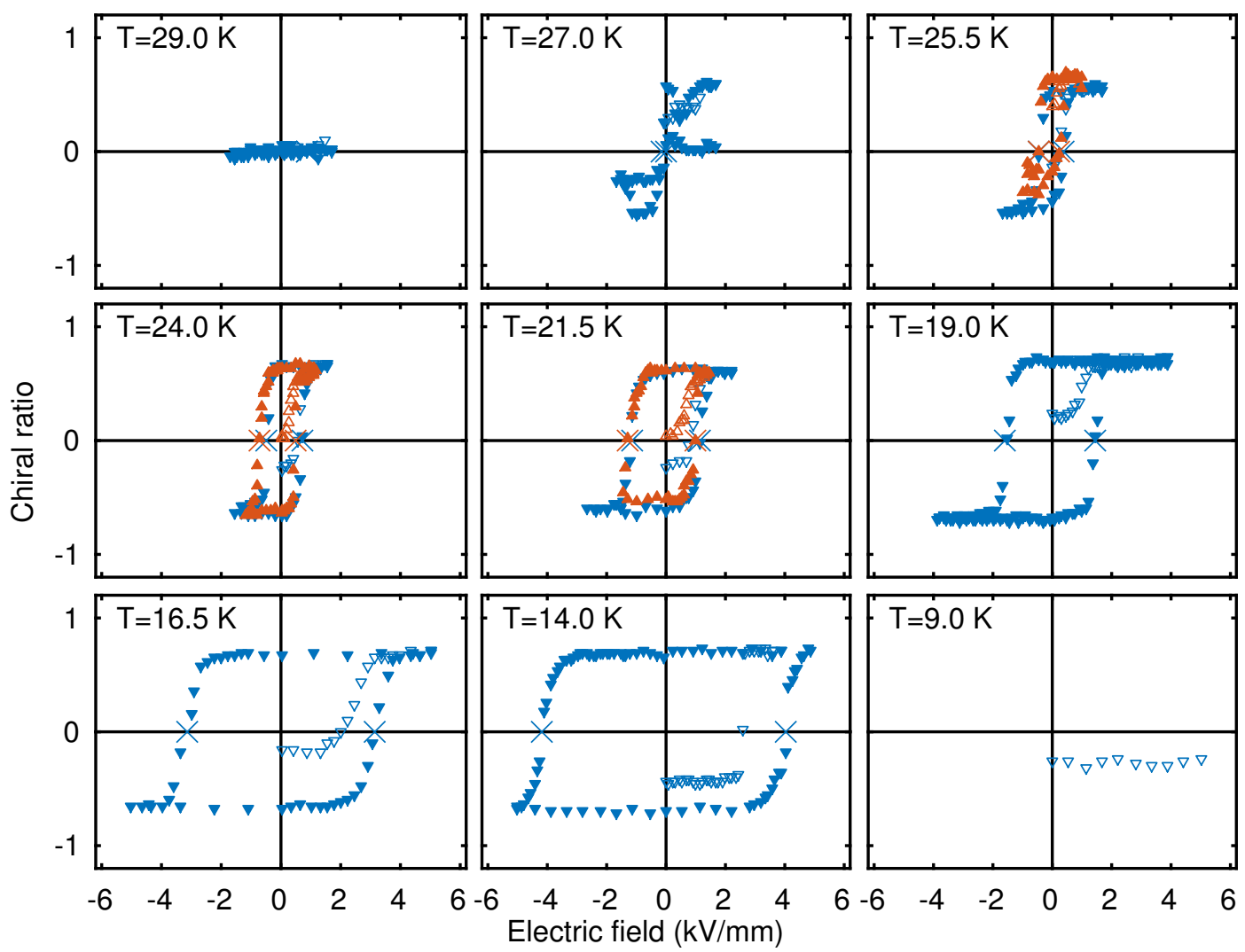

Figure 4. (Color online) Hysteresis loops in $\mathrm{TbMnO}_{3}$ obtained by measuring the chiral ratio as a function of external electric field at different temperatures for two different samples at POLI-HEIDI (thicknesses of 0.9 and $3.1 \mathrm{~mm}$ ). Open triangles denote the data taken immediately after cooling and filled symbols denote the data taken to characterize the full hysteresis loops. Triangles plotted in orange show the three loops taken with the thicker crystal. Crosses denote the coercive field values. The loops were recorded after zero-field cooling from $50 \mathrm{~K}$.

furthermore is not a perfect cycloid [25]. With the elliptical magnetic structure we calculate an ideal mono-domain chiral ratio $r_{\text {chir }}= \pm 0.93$ for $\vec{Q}=\left(\begin{array}{lll}2 & 0.28 & 1\end{array}\right)$.

The effect of small electric fields applied during cooling from the paramagnetic into the chiral magnetic phase is depicted in Figure 3. The sequence was measured with reducing and alternating field strengths. Very small fields of a few tenths of $\mathrm{V} / \mathrm{mm}$ applied upon cooling are sufficient to produce finite preference for one chiral domain. However, saturation is not reached for such small fields. Instead, the chiral ratio shows a linear dependence on the electric field applied during the cooling, at least in this lowfield range. Note, however, that the chiral response of the samples is asymmetric with respect to the applied field, as it even exhibits finite values for zero electric field.

In the following we focus on the possibility to reverse chiral magnetic domains by varying the electric field at constant temperature. This represents the multiferroic hysteresis loops, i.e. the control of magnetic order by an external electric field. The obtained hysteresis loops are shown in Figure 4. All hysteresis loops were recorded after 

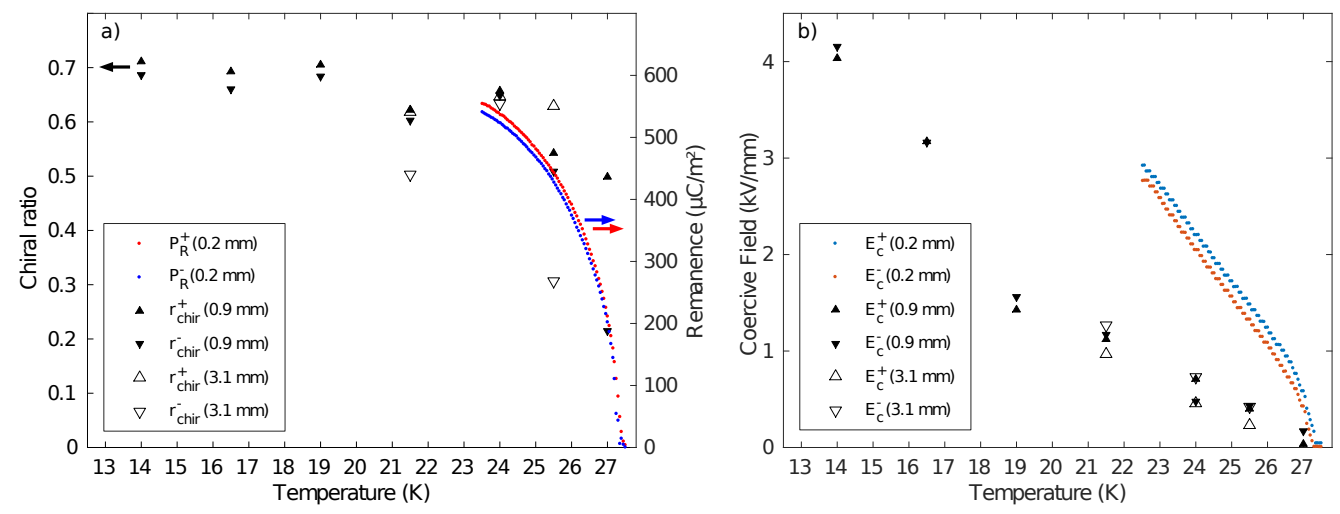

Figure 5. (Color online) (a) Temperature dependence of the remanent ferroelectric polarization in $\mathrm{TbMnO}_{3}$ as obtained from the dielectric hysteresis loops shown in Fig. 6. In comparison we also show the temperature dependence of the remanent chiral ratios observed in polarized neutron diffraction. In both parts larger triangles correspond to the neutron results, and the tip of the triangle indicates the direction of the applied electric fields. (b) Temperature dependence of the coercive fields obtained in the neutron diffraction and dielectric measurements. The positive and negative coercive fields obtained from the chiral-ratio loops match perfectly underlining the symmetric character of the sample aside from the results obtained for the thicker crystal with neutron diffraction. Furthermore, the coercive fields obtained in the neutron experiment do not depend on sample thickness. The coercive field obtained from the dielectric experiments are considerably higher.

zero-field cooling from $50 \mathrm{~K}$ to the respective temperature. To ensure that there was no electric field applied during cooling the sample surfaces were short-circuited for most of the loops shown in Figure 4. Only the curve taken at $19 \mathrm{~K}$ was obtained after cooling the sample with open contacts, which seems to induce a different direction of preference. It is a remarkable finding that the initial curves always start at finite values even for cooling with short-circuited surface contacts. The hysteresis loops were recorded by varying the electric field in finite steps and by recording the neutron intensities at constant fields. Due to the limited count rates, it was necessary to count several minutes per point. Therefore, the shown hysteresis loops are quasi-static for relaxation times of the order of minutes and below.

Most recorded hysteresis loops show a symmetric shape. Both the coercive fields and the saturation values are in good accordance between the two directions. The coercive field increases with decreasing temperature while an approximately constant saturation value of $r_{\text {chir }}$ can be reached at low temperatures by applying a high enough field, see Fig. 5(a) and (b). Note, however, that the chiral ratio describes the domain population multiplied with the intrinsic chiral fraction of the magnetic scattering and, therefore, it is not expected to continuously increase upon cooling while the ferroelectric polarization follows $\vec{S}_{i} \times \vec{S}_{j}$. The domain dynamics of the sample becomes stiffer when cooling deeper into the multiferroic phase. This behavior qualitatively resembles the results reported for $\mathrm{MnWO}_{4}$ [17, 18]. At $9 \mathrm{~K}$ the domains are strongly pinned; therefore, 
the electric field causes an electrical breakdown before the chiral ratio can be affected. Already at $14 \mathrm{~K}$ the maximal applicable electric field hardly surpasses the coercive field needed to start reverting the multiferroic domains within the time scales of the experiment.

The hysteresis loops of the two samples of different thicknesses match well each other concerning the coercive fields aside from a more pronounced asymmetry for the thicker crystal. In order to apply the same electric field strength to the thicker sample a larger voltage has to be applied. For that reason it is not possible to reach as high electric fields for the thicker sample as for the thinner sample. Therefore, we registered only three hysteresis loops at higher temperatures for the sample, which was $3.1 \mathrm{~mm}$ thick. The coercive fields for all temperatures are shown in Figure 5 (b). The positive and negative coercive fields match well underlining the symmetric character of the thinner samples. The coercive field shows roughly a linear dependence on the temperature. There is less agreement concerning the remanent values of the chiral ratio observed in the two samples of different thickness. The thicker sample of the neutron experiment remains less polarized.

\subsection{Ferroelectric hysteresis loops detected by capacitor measurements}

The ferroelectric polarization of $\mathrm{TbMnO}_{3}$ was studied macroscopically with a modified Sawyer-Tower circuit. The ferroelectric polarization $P$ versus electric field $E$ hysteresis loops obtained by averaging four successive cycles are shown in Fig. 6 for different temperatures. The hysteresis loops are not fully flat in their saturation region due to the dielectric background contribution, as it is usually observed in ferroelectrics, and due to finite conductivity effects. Therefore we have substracted a loop taken in the paraelectric phase from all the other loops. Finite slopes then still arise from the minor differences in conductivity and dielectric constant occurring with the variation of the temperature. For clarity we show only the data taken in $0.2 \mathrm{~K}$ temperature steps, but intermediate temperatures were also measured.

The hysteresis loops in Fig. 6 show that the ferroelectric transition sets in at 27.3(1) $\mathrm{K}$ in perfect agreement with the susceptibility data and literature. Below this temperature we find remanent polarization as well as finite width and area in the loops. The slight change in the slope of the entire loop can be attributed to the maximum of the dielectric constant at $T_{C}$ [4].

The area of the loop considerably increases upon cooling. The remanent polarization is almost identical to the saturation polarization after correcting for the finite slope. The temperature dependence of the remanent polarization matches the temperature dependence of the polarization measured by the pyrocurrent method [4, 24] as long as temperatures stay above $24 \mathrm{~K}$, see Fig. 5 and 6 . This indicates that for temperatures larger than $24 \mathrm{~K}$ the polarization can be fully controlled with the maximum applied electric field of $3 \mathrm{kV} / \mathrm{mm}$ and within a time of about one second. In contrast the data taken below $24 \mathrm{~K}$ show that saturation polarization cannot be reached indicating 


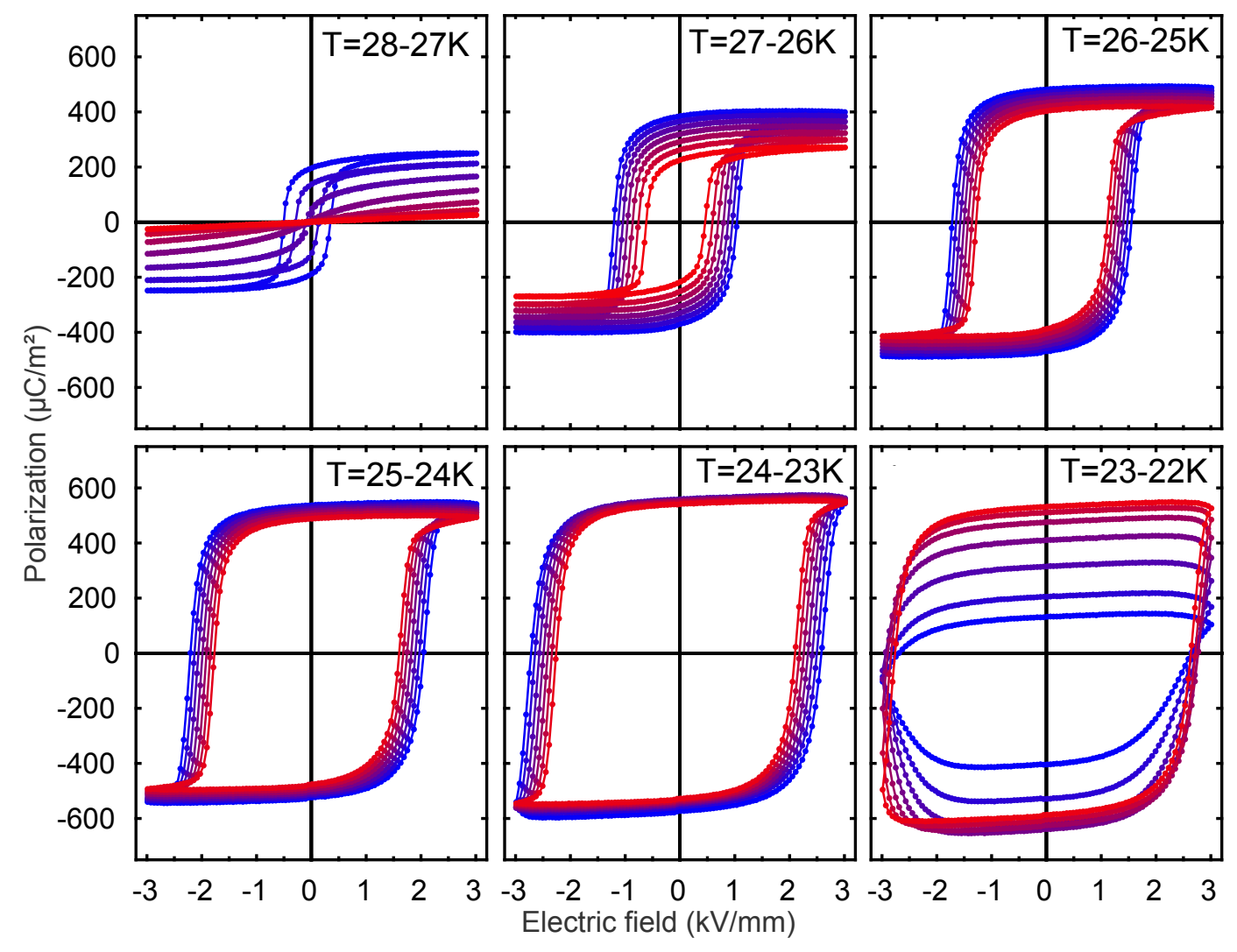

Figure 6. (Color online) Dielectric hysteresis loops recorded by a modified SawyerTower circuit on a $0.2 \mathrm{~mm}$ thick sample. The colors indicate the temperature varied within each panel in $0.2 \mathrm{~K}$ steps from the colder (blue) to the warmer (red) temperatures. A linear background determined above the ferroelectric transition was substracted.

that the relaxation times surpass the times of the measurement. Between 23 and $24 \mathrm{~K}$ one may recognize that saturation is not reached before the field starts to decrease from its maximal values, and below $23 \mathrm{~K}$ the polarization continues to increase in absolute size even when the electric field is already decreasing. This clearly shows that the measurement was too fast to allow the $\mathrm{TbMnO}_{3}$ domains to fully relax. Note that the neutron experiment due to the low count rates was sufficiently slow to allow for full relaxation to lower temperatures.

The temperature dependence of the coercive field is shown in Fig. 5(b). In this dielectric experiment there is no significant difference in the coercive fields obtained for the two field directions, which agrees to the neutron experiments and also to the symmetric values of the remanent polarization. In the studied temperature interval the coercive field increases linearly with temperature after a small jump at the transition. Compared to the neutron study the coercive fields are considerably larger in spite of the fact that all the crystals were cut from the same large single crystal. However, the much thinner sample used in the dielectric study can more strongly suffer from the cutting process. In addition fatigue effects can be stronger in the sample used for 
the macroscopic measurement as it went through a much larger number of thermal and electrical cycles before the shown experiments were performed. Coercive fields in $\mathrm{TbMnO}_{3}$ are seem to be sample dependent.

\section{Conclusions}

In conclusion we have shown by polarized neutron diffraction and by macroscopic measurements that the population of multiferroic domains in bulk $\mathrm{TbMnO}_{3}$ can be controlled through a moderate electric field of the order of several $\mathrm{kV} / \mathrm{mm}$. Neutron diffraction determines the reversion of the chiral magnetism while the macroscopic measurement senses the ferroelectric polarization. The coercive fields in both studies are of the same order but significant differences can be ascribed to differences in the samples used. The chiral magnetic and polarization hysteresis loops confirm once more the close coupling of the two properties.

\section{Acknowledgments}

This work was supported through the Bundesministerium für Bildung und Forschung through project 05K13PK1 and 05K10PA2. We are thankful to W. Luberstetter for the technical support during the neutron diffraction experiment and to S. Masalovich for supplying ${ }^{3} \mathrm{He}$ spin-filter cells.

[1] W. Eerenstein, N.D. Mathur, J.F. Scott, Nature 442759 (2006).

[2] S.-W. Cheong, M. Mostovoy, Nature Mater. 613 (2007).

[3] H. Katsura, N. Nagaosa, A.V. Balatsky, Phys. Rev. Lett. 95, 057205 (2005); M. Mostovoy, ibid. 96, 067601 (2006); I.A. Sergienko, E. Dagotto, Phys. Rev. B 73, 094434 (2006).

[4] T. Kimura, T. Goto, H. Shintani, K. Ishizaka, T. Arima, Y. Tokura, Y., Nature 42655 (2003).

[5] G. Lawes, A.B. Harris, T. Kimura, N. Rogado, R.J. Cava, A. Aharony, O. Entin-Wohlman, T. Yildirim, M. Kenzelmann, C. Broholm, A.P. Ramirez, Phys. Rev. Lett. 95087205 (2005).

[6] K. Taniguchi, N. Abe, T. Takenobu, Y. Iwasa, T. Arima, Phys. Rev. Lett. 97097203 (2006); O. Heyer, N. Hollmann, I. Klassen, S. Jodlauk, L. Bohatý, P. Becker, J.A. Mydosh, T. Lorenz, D. Khomskii, J. Phys.: Cond. Matter 18 L471 (2006); A.H. Arkenbout, T.T.M. Palstra, T. Siegrist, T. Kimura, Phys. Rev. B 74184431 (2006).

[7] T. Arima, J. Phys. Soc. Jpn., 76073702 (2007).

[8] T. A. Kaplan and S. D. Mahanti, Phys. Rev. B 83, 174432 (2011).

[9] T. Nakajima, S. Mitsuda, S. Kanetsuki, K. Prokes, A. Podlesnyak, H. Kimura, and Y. Noda, J. Phys. Soc. Jpn. 76, 043709 (2007).

[10] M. Soda, K. Kimura, T. Kimura, M. Matsuura, and K. Hirota, J. Phys. Soc. Jpn. 78, 124703 (2009).

[11] M. Kenzelmann, G. Lawes, A. B. Harris, G. Gasparovic, C. Broholm, A. P. Ramirez, G. A. Jorge, M. Jaime, S. Park, Q. Huang, A. Ya. Shapiro, and L. A. Demianets, Phys. Rev. Lett. 98, 267205 (2007).

[12] M. Baum, J. Leist, Th. Finger, K. Schmalzl, A. Hiess, L. P. Regnault, P. Becker, L. Bohatý, G. Eckold, and M. Braden Phys. Rev. B 89, 144406 (2014).

[13] M. Bibes and A. Barthélémy, nature materials 7, 425 (2008). 
[14] D. Meier, N. Leo, M. Maringer, Th. Lottermoser, M. Fiebig, P. Becker, and L. Bohatý, Phys. Rev. B 80, 224420 (2009).

[15] D. Meier, M. Maringer, Th. Lottermoser, P. Becker, L. Bohatý, and M. Fiebig Phys. Rev. Lett. 102, 107202 (2009).

[16] P.J. Brown, Spherical Neutron Polarimetry, in Neutron Scattering from Magnetic Materials, Edt. T. Chatterji, Elsevier B.V., Amsterdam (2006).

[17] M. Baum, A.C. Komarek, S. Holbein, M.T. Fernández-Díaz, G. André, A. Hiess, Y. Sidis, P. Steffens, P. Becker, L. Bohatý, and M. Braden, Phys. Rev. B 91, 214415 (2015).

[18] T. Finger, D. Senff, K. Schmalzl, W. Schmidt, L.P. Regnault, P. Becker, L. Bohatý, M. Braden, Phys. Rev. B 81054430 (2010).

[19] Y. Yamasaki, H. Sagayama, T. Goto, M. Matsuura, K. Hirota, T. Arima, and Y. Tokura, Phys. Rev. Lett. 98, 147204 (2007).

[20] S. Seki, Y. Yamasaki, M. Soda, M. Matsuura, K. Hirota, and Y. Tokura, Phys. Rev. Lett. 100, 127201 (2008).

[21] H. Sagayama, K. Taniguchi, N. Abe, T. H. Arima, M. Soda, M. Matsuura, and K. Hirota, Phys. Rev. B 77, 220407(R) (2008).

[22] A. Poole, P. J. Brown, and A. S. Wills, J. Phys.: Conf. Ser. 145, 012074 (2009).

[23] M. Baum, K. Schmalzl, P. Steffens, A. Hiess, L. P. Regnault, M. Meven, P. Becker, L. Bohatý, and M. Braden, Phys. Rev. B 88, 024414 (2013).

[24] T. Goto, T. Kimura, G. Lawes, A. P. Ramirez, and Y. Tokura, Phys. Rev. Lett. 92, 257201 (2004).

[25] M. Kenzelmann, A. B. Harris, S. Jonas, C. Broholm, J. Schefer, S. B. Kim, C. L. Zhang, S.-W. Cheong, O. P. Vajk, and J.W. Lynn, Phys. Rev. Lett. 95, 087206 (2005).

[26] T. Kimura, G. Lawes, T. Goto, Y. Tokura, and A. P. Ramirez, Phys. Rev. B 71, 224425 (2005).

[27] N. Aliouane, K. Schmalzl, D. Senff, A. Maljuk, K. Prokes, M. Braden, and D. N. Argyriou, Phys. Rev. Lett. 102, 207205 (2009).

[28] A. Glavic, C. Becher, J. Voigt, E. Schierle, E. Weschke, M. Fiebig, and T. Brückel, Phys. Rev. B 88, 054401 (2013).

[29] V. Hutanu, M. Meven, E. Lelièvre-Berna, and G. Heger, Physica B 404, 2633 (2009).

[30] V. Hutanu, M. Meven, S. Masalovich, G. Heger, and G. Roth, J. Phys.: Conf. Ser. 294, 012012 (2011).

[31] F. Tasset and E. Ressouche, Nucl. Instr. Meth A 359, 537 (1995). 